\title{
Controlling the growth of Metal-Organic Frameworks using different gravitational-forces
}

\author{
Joseph J. Richardson, ${ }^{[a]}$ Kang Liang, ${ }^{[a]}$ Fabio Lisi, ${ }^{[b]}$ Mattias Björnmalm, ${ }^{[c]}$ Matthew Faria, ${ }^{[c]}$ Junling \\ Guo, ${ }^{[c]}$ Paolo Falcaro*[d]
}

Abstract: Control over Metal-Organic Framework (MOF) size and morphology is interesting for both fundamental and applied science. Gravitational-force $(g)$ is generally acknowledged as an interesting parameter for controlling crystal size, however a dedicated study on the effect of $g$ on MOF synthesis is missing. Here, we investigate the effect of varied $g(<1,1,20,50$, and 100$)$ during the crystallization of different MOFs (ZIF-8, Tb 2 (BDC) $)_{3}$ and HKUST-1) in solution. The obtained MOFs were investigated using dynamic light scattering (DLS), X-ray scattering (SAXS and WAXS), and scanning electron and optical microscopy (SEM and OM, respectively). When compared with standard-g $(g=1)$, high- $g(g=20)$ gave rise to the formation of smaller MOF crystals, while low- $g(g<1)$ led to larger crystals likely due to facet-oriented crystal fusion. This demonstrates that gravity and $g$-force can be used to rationally control the size of different MOFs by increasing or decreasing convection (mass transfer) and sedimentation

\section{Introduction}

Metal-Organic Frameworks (MOFs), also called Porous Coordination Polymers (PCP), are crystalline porous materials prepared by connecting inorganic nodes with bridging ligands. Their exceptional accessible surface area, up to $7,000 \mathrm{~m}^{2} \mathrm{~g}^{-1}$ and the modular construction, enable their exploitation for a number of different applications, ${ }^{[1]}$ including gas storage,,$^{[2]}$ separation, ${ }^{[3]}$ catalysis, ${ }^{[4]}$ sensing, ${ }^{[5]}$ microelectronics, ${ }^{[6]}$ optics, ${ }^{[7]}$ drug delivery and biotechnology, ${ }^{\left[{ }^{[]}\right.}$forensics, ${ }^{[9]}$ and environmental remediation. ${ }^{[10]}$ Different methodologies have been proposed for controlling MOF properties including postsynthetic modification, ${ }^{[11]}$ incorporation of inorganic and/or biological components, ${ }^{[9,}$, 12$]$ or control of particle size and morphology. ${ }^{[13]}$ Interestingly, the latter strategy does not require the modification of the MOF using chemical reactions or encapsulated extrinsic functionalities. The precise tuning of MOF

[a] J. J. Richardson, K. Liang

Manufacturing, CSIRO, Clayton, Victoria, Australia

E-mail: jj.richardson@csiro.au

[b] F. Lisi

School of Chemistry and Bio21 Institute, University of Melbourne

Parkville, Victoria, Australia

[c] M. Björnmalm, M. Faria, J. Guo

ARC Centre of Excellence in Convergent Bio-Nano Science and Technology, and the Department of Chemical and Biomolecular Engineering, The University of Melbourne, Parkville, Victoria, Australia

[d] P. Falcaro

Graz University of Technology, Institute of Physical and Theoretical Chemistry, Stremayrgasse 9, $8010 \mathrm{Graz}$, Austria

E-mail: paolo.falcaro@tugraz.at

Supporting information for this article is given via a link at the end of the document crystal size and shape influences the accessible surface area and diffusion properties of the final porous material. ${ }^{[14]}$ Additionally, this control allows for the use of single MOF crystals as building blocks for the fabrication of hierarchical structures. ${ }^{[15]}$

To date, the control of crystal morphology has been achieved using coordination modulators, surfactants, ${ }^{[16]}$ amino acids, ${ }^{[17]}$ seeds, ${ }^{[18]}$ and post chemical etching. ${ }^{[19]}$ Notably, a variable that has been historically known to influence crystal formation in general is gravity and gravitational-force $(g)$, where high-g induces the formation of smaller crystals due to increased sedimentation, and low-g leads to larger crystals due to a decrease in convection (heat and mass transfer) and related convective mixing. ${ }^{[20]}$ However, few crystallization processes have been studied across the board at low, normal and high- $g$ due to the general experimental and technical difficulty surrounding common crystallization procedures, i.e., complex equipment, varied solvents, high temperatures, low or high pressures, etc. ${ }^{[20 c]}$ In terms of MOFs, a preliminary study proposed by $\mathrm{Hu}$ et al. ${ }^{[21]}$ demonstrated that extremely highspeed centrifugation (3000-5000 RPM) leads to the formation of amorphous nanoscale MOF seeds. Inspired by that work and the decades of previous research on the effects of microgravity on crystal formation, we examined the formation of different MOFs under varied $g$. Deeply characterized MOFs, namely zeolitic imidazolate framework-8 (ZIF-8), ${ }^{[22]}$ HKUST-1 $\left(\mathrm{Cu}_{3}(\mathrm{BTC})_{2}\right){ }_{,}{ }^{[23]}$ and $\mathrm{Tb}_{2}(\mathrm{BDC})_{3},{ }^{[24]}$ were chosen as candidates for this experiment as they can form rapidly under mild conditions without the need for hazardous solvents. To examine the effects of $g$ on the crystallization of these MOFs, diverse experimental processes were developed (Figure 1). A slow rotor centrifuge capable of spinning as low as 20 RCF was used to generate high- $g$ within 2 orders of magnitude of standard- $g$, namely 20,50 , and $100 \mathrm{~g}$. Low-g was generated using two novel experimental protocols not widely present in the literature. Short periods (1-3 s) of low- $g$ were generated during the crystallization process by a manual drop from a $\sim 25 \mathrm{~m}$ building. This has advantages over drop towers as it was not necessary to construct complicated experimental apparatus or secure access to specialized facilities, although some downsides such as increased drag and reduced experimental controls existed. ${ }^{[20 c, 25]}$ Prolonged periods (10-15 s) of low- $g$ were generated by skydiving out of an airplane $(\sim 4,000 \mathrm{~m})$, with the use of a parachute to minimize the gravitational challenges after freefall (Video S1). ${ }^{[26]}$ This method has advantages over parabolic flights and international space station (ISS) experiments as those procedures are extremely expensive (from tens of thousands to tens of millions of dollars) and lengthy, especially for ISS allocations. ${ }^{[27]}$ Using these unique and accessible experimental protocols, and maintaining stable temperatures during crystallization, it was found that lower $g$ results in larger MOF crystals, while higher-g results in smaller MOF crystals. 
A
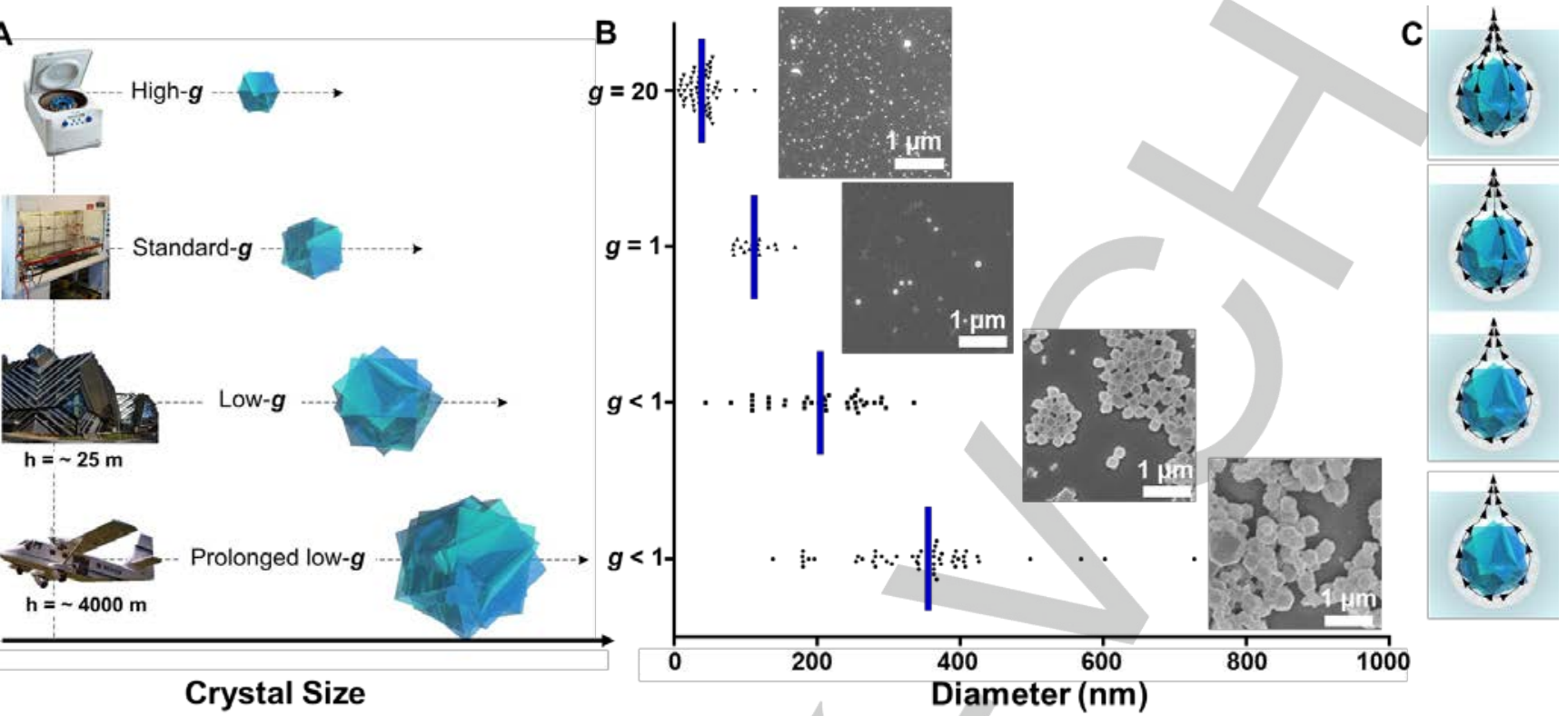

Crystal Size

Figure 1. Different $g$-force effects on the crystallization of MOFs. A, scheme of the experiments performed where during crystallization, the MOF precursors were exposed to varying $g$ using different experimental protocols, with lower $g$ resulting in larger crystals. High- $g(g=20-100)$ was generated using a centrifuge. Normal- $g(g=1)$ was the result of standard static synthesis on a benchtop. Low- $g(g<1)$ was generated for a short period of time $(2-3$ s) by dropping the sample off of $\mathrm{a} \sim 25 \mathrm{~m}$ roof just after mixing the precursors and for prolonged periods (10-15 s) by skydiving from just after mixing the precursors. $\mathrm{B}$, plot of the average diameter (blue bar) and the crystal size distribution (black dots) of ZIF-8 MOFs prepared under different $g$ accompanied by representative SEM images C, Nanoscale-convection during crystal growth at varying $g$, where depletion zones (white) appear as the bulk precursor solution (blue) gets consumed. Subsequently, the lower density water of the depletion zones rises due to gravity, resulting in convective mixing as established in the literature. Note this is not an exhaustive scheme of all the mechanisms leading to convection.

\section{Results and Discussion}

\section{Identification of MOF protocols suitable for experiments at} different $g$

In normal laboratory conditions under standard-g $(g=1)$, dynamic light scattering (DLS) was used to determine the kinetics governing the nucleation/growth, and the concentration of the precursors needed for the rapid crystallization of our MOFs. This was crucial, as low-g can only be obtained for short periods of time in our case (maximum tens of seconds), while high-g requires a rotor to spin up to speed and therefore requires longer growth processes (minimum tens of seconds). Because of these time-constraints, diverse crystallization times were desired and engineered for the three different MOFs. For $\mathrm{Tb}_{2}(\mathrm{BDC})_{3}$, after mixing aqueous solutions of $20 \mathrm{mM}$ for each precursor, the growth of the crystals was extremely rapid with a plateau occurring within $<10 \mathrm{~s}$ (Figure S1). This suggested that $\mathrm{Tb}_{2}(\mathrm{BDC})_{3}$ would only be suitable for low- $g$ experiments. Due to the high aspect ratio of the MOF crystals, it was not possible to determine the exact size and kinetics solely from DLS data. For the preparation of ZIF-8, serine ${ }^{[17]}$ was used as a cocrystallization agent (final concentration $1.3 \mathrm{mg} \mathrm{mL}^{-1}$ ) in order to induce the ZIF-8 formation within the short timescale suitable for low-g studies (<20 s). Aqueous solutions of the ZIF-8 precursors (160 mM n-methyl imidazole and $40 \mathrm{mM}$ zinc) were mixed at equal volumes, and we found that the majority of ZIF-8 formed in $\sim 20 \mathrm{~s}$. In the subsequent $40 \mathrm{~s}$, the ZIF-8 crystal size stabilized in the $130-160 \mathrm{~nm}$ range, suggesting that ZIF-8 would be suitable for high-g and low- $g$ experiments (Figure S2). For HKUST-1, we found that a final solution of $75 \%$ ethanol with final concentrations of $50 \mathrm{mM}$ for copper and $37 \mathrm{mM}$ for BTC induced the formation of sub-micron seeds within $10 \mathrm{~s}$. The count rate spiked in the first minute as nucleation occurred, and then linearly decreased as the crystals both fused and sedimented. ${ }^{[29]}$ Simultaneously the growth of the crystals proceeded until the particles were too big for DLS detection (>3 $\mu \mathrm{m}$ at $20 \mathrm{~min}$ ), suggesting that HKUST-1 was also suitable for high and low- $g$ experiments (Figure S3).

\section{High-g experiments}

The high- $g$ portion of the study was conducted using HKUST-1 and ZIF-8 as their growth kinetics were slow enough to negate the time-lag in spinning the centrifuge up to speed. In both cases, the synthesis of MOFs performed at high- $g$ was compared with a control sample prepared at normal $g$, where the same protocols were used, i.e., precursor concentrations, reaction time and temperature. Using scanning electron microspcopy (SEM) and optical microscopy, we found that high-g reduced the crystal size of HKUST-1 when compared to standard-g (Figure 2). Increasing the $g$ from 20 to 50 or 100 did not make any 
substantial observable difference (Figures S4 and S5). This result suggested that the MOF particles were being sedimented and collected rapidly, ${ }^{[21]}$ even at $20 \mathrm{~g}$, and that the increased precursor transport ${ }^{[30]}$ from improved convective mixing ${ }^{[31]}$ was helping to terminate crystal growth and fusion. ${ }^{[20 \mathrm{~d}]}$ Specifically, at $20 \mathrm{~g}$ the HKUST-1 was $6 \times$ smaller than standard- $g$ (mean diameter of $53.9 \mu \mathrm{m}$ vs $9.1 \mu \mathrm{m}$ ), although the diffraction pattern and shape were similar at standard and high $g$ (Figure 2 and Figure S5). This is similar to what has been seen with different sized ZIFs prepared under varying kinetic regimes. ${ }^{[30]}$ The ZIF-8 crystals prepared with a burst of $10 \mathrm{~s}$ of $20 \mathrm{~g}$ and left to sit for 10 min, could not be readily pelleted with standard centrifugation $(\sim 10,000 \mathrm{RCF})$ due to their small size, however some small particles were still recovered and investigated with SEM (Figure 1 and Figure S6). Specifically, these ZIF-8 particles were $3 \times$ smaller than those prepared at standard- $g$ (mean diameter of 38 $\mathrm{nm}$ vs $120 \mathrm{~nm}$ ). Collectively, these high- $g$ data suggested that increased convection was experienced with increased $g,{ }^{[31]}$ leading to smaller crystals. Like the previous report on growing MOFs under extremely high centrifugation, ${ }^{[21]}$ our MOFs were smaller when exposed to high-g, although they remained crystalline unlike the previous MOF study. The preserved crystallinity can be reconciled by the fact that our experiments were conducted at significantly lower centrifugation speed.

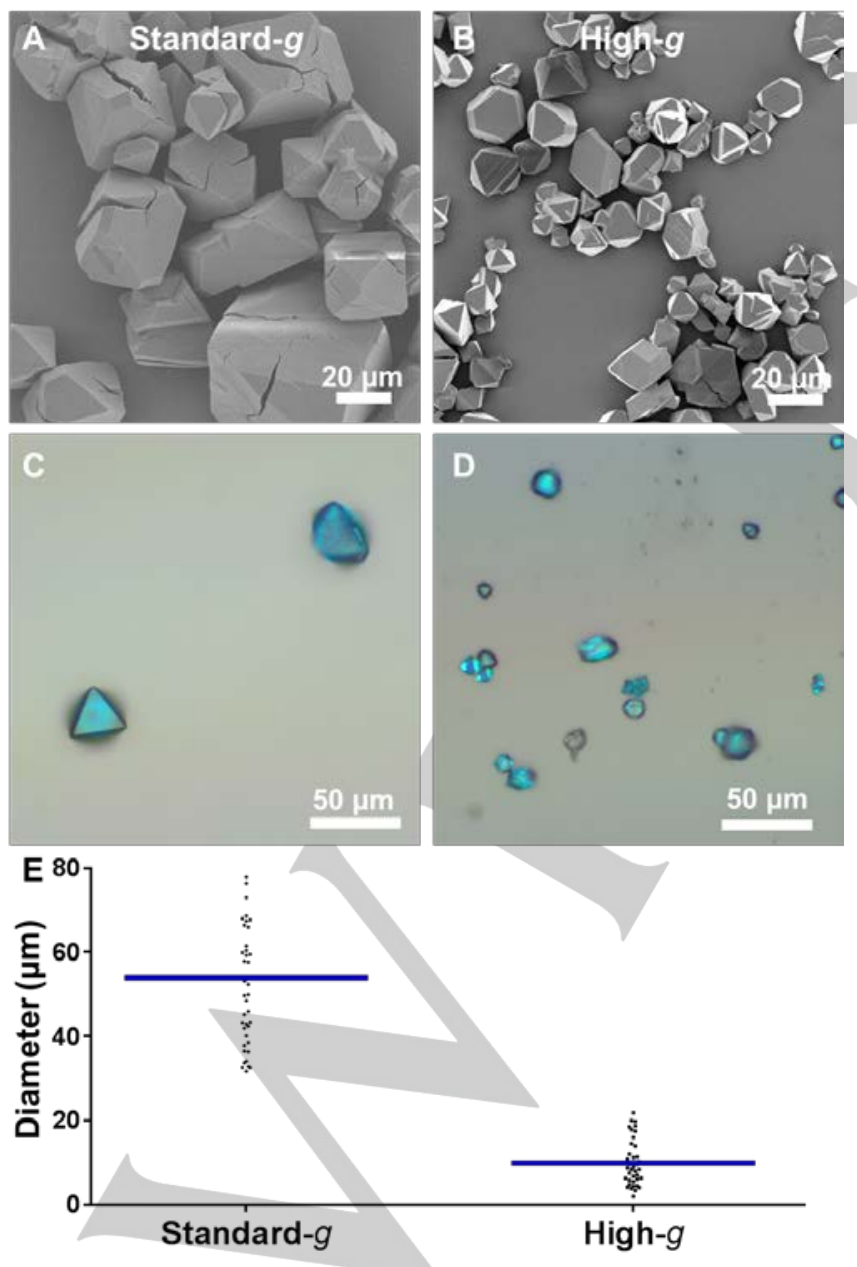

Figure 2. The effect of high-g on HKUST-1 growth. SEM $(A, B)$ and optical images $(C, D)$ of the HKUST-1 prepared at standard- $g(A, C)$ and high- $g(B, D)$ $\mathrm{E}$, graph of average diameter (blue bar) and size distribution (black dots) depending on the $g$ applied during synthesis. HKUST-1 was prepared overnight by mixing the precursors and then placing the samples either on a benchtop (standard-g), or into a centrifuge maintained at 20 RCF $(20 \mathrm{~g})$ (highg).

\section{Low-g experiments}

The high- $g$ results confirmed the influence of $g$ on MOF growth. To further elucidate mechanistic trends, a low- $g$ portion of the study was conducted with $\mathrm{Tb}_{2}(\mathrm{BDC})_{3}, \mathrm{ZIF}-8$ and HKUST-1 . Firstly, a drop experiment was conducted, and accelerometer readings generated during the fall from $\sim 25 \mathrm{~m}$ showed that $\sim 2-3$ $\mathrm{s}$ of low- $g$, with a lowest absolute acceleration of $2 \mathrm{~m} \mathrm{~s}^{-2}$, was experienced during the drop (Figure $\mathbf{3}$ and Figure S7). Although this low- $g$ time period was short, the kinetics of MOF formation was fast enough to allow for differences in crystal growth. As postulated, the drop experiments showed a marked size increase for the HKUST-1 and ZIF-8 (Figure 1, 3 and Figure S8). However no difference was observed for the $\mathrm{Tb}_{2}(\mathrm{BDC})_{3}$. Specifically, both the HKUST-1 and the ZIF-8 were roughly $2 \times$ larger when formed in low- $g$, (mean diameter of $1.1 \mu \mathrm{m}$ vs 0.52 $\mu \mathrm{m}$ for HKUST-1 and $200 \mathrm{~nm}$ vs $120 \mathrm{~nm}$ for ZIF-8).

\section{Prolonged low- $g$ experiments}

Encouraged by the low-g data generated with the drop experiments, prolonged low- $g$ was generated using skydiving to investigate the $\mathrm{ZIF}-8$ and $\mathrm{Tb}_{2}(\mathrm{BDC})_{3}$, while HKUST-1 precursors could not be brought on the plane due to the presence of ethanol. The skydiving jump experiment required a home-built injector that allowed the solutions to be mixed immediately before exiting the plane (Video S1 and Figure S9), and a mobile lab that allowed the samples to be washed immediately after landing (Figure S10). Accelerometer data gathered during the jump showed that prolonged low-g (10-15 s) was reached, with the lowest absolute acceleration being $4 \mathrm{~m} \mathrm{~s}^{-2}$ (Figure 1, 4 and Figures S11, S12). The prolonged low-g resulted in substantially larger MOFs than both standard- $g$ and the dropped low-g samples for ZIF-8 (Figure 1). This suggested that the length of time below $1 \mathrm{~g}$ was more important than the absolute acceleration reached. Interestingly, the $\mathrm{Tb}_{2}(\mathrm{BDC})_{3}$ replicates not only had larger crystals than those at standard- $g$, but each replicate also had different unique morphologies of giant $(>20$ $\mu \mathrm{m})$ fused crystals in complex ball or claw shapes (Figure S13) Specifically, the prolonged low- $g$ ZIF-8 and $\mathrm{Tb}_{2}(\mathrm{BDC})_{3}$ were both larger than standard- $g$ crystals (mean diameter of $630 \mathrm{~nm}$ (replicate 1) and $355 \mathrm{~nm}$ (replicate 2) vs $120 \mathrm{~nm}$ for ZIF-8 and mean length of $1.4 \mu \mathrm{m}$ vs $3.2 \mu \mathrm{m}$ for $\left.\mathrm{Tb}_{2}(\mathrm{BDC})_{3}\right)$. This aspect might elicit further investigation. 

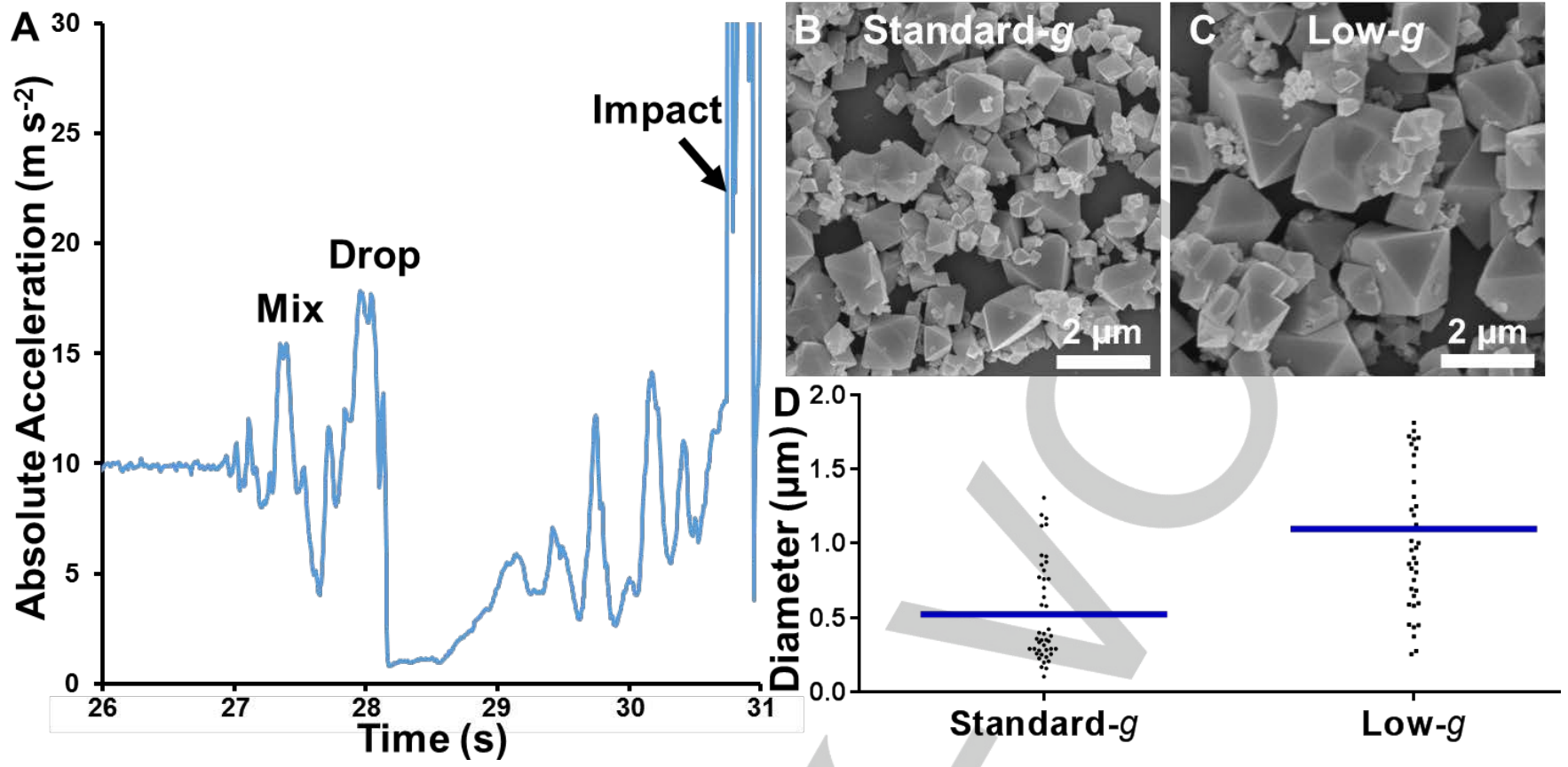

Figure 3. Effect of short periods of low-g (2-3 s) on HKUST-1 growth. A, processed accelerometer data for the drop experiment showing the acceleration experienced at each time point. SEM image of the HKUST-1 prepared under standard- $g$ (B) and low- $g$ (C). D, graph of average diameter (blue bar) and size distribution (black dots) depending on the $g$ applied during the synthesis. HKUST-1 was prepared by mixing the precursors and then placing the samples either on a benchtop (standard- $g$ ), or dropping them off of a $\sim 25 \mathrm{~m}$ building (low- $g$ ).

The combined low-g and prolonged low-g data is consistent with the literature on low- $g$ colloidal nucleation and crystallization. ${ }^{[20 c]}$ For example, other colloidal nucleation processes, such as the crystallization of polymer particles have shown that the reaction proceeds significantly quicker and with higher fidelity in low- $g{ }^{[32]}$ In low- $g$, the transport process is only governed by diffusion and is further reduced than standard-g synthesis as mixing due to convection is greatly reduced; therefore the predominant driving force of monomer rearrangement becomes further favored at low-g. ${ }^{[30]}$ However, rearrangement of single monomers and ligand-metal cluster replacement ${ }^{[33]}$ is unlikely due to depletion zones that arise due to the lack of convective mixing in low- $g$, and rather the rearrangement of small crystals collectively, i.e., fusion of crystals along facets becomes favorable. ${ }^{[29]}$ This can be clearly seen in the SEM images of the low- $g$ samples where fused crystals are apparent for all three MOFs, with far less fusion in the standard- $g$ and high-g samples. This makes Ostwald ripening less likely as a growth mechanism for these three MOFs, as smaller particles are still visible in the images. Similarly, the polydispersity increases as $g$ decreases, suggesting that the separate regimes of nucleation and growth become merged in low- $g$, as the overlapping of these regimes has been previously shown to increase the polydispersity and increase the particle size of ZIF-8 (Figure 1). ${ }^{[28,30]}$ Additionally, it is well known that low- $g$ reduces the likelihood of crystal nucleation due to the decrease in convective mixing, ${ }^{[20 \mathrm{~d}]}$ which allows the growth regime to overlap nucleation. Slower nucleation is also known to increase polydispersity in MOFs. ${ }^{[34]}$ Furthermore, polydispersity can increase in low-mixing situations on short time scales due to local concentration differences in the solution allowing some crystals to access more MOF precursors than others and therefore grow larger. ${ }^{[20 \mathrm{~d}]}$ A final interesting point is that the average diameters or lengths scaled by the same factors between the different MOFs exposed to similar low- $g$. Specifically, low- $g$ increased the size by a factor of 2 for both the HKUST-1 and ZIF-8, while prolonged low- $g$ increased the size by a factor of 3 for both ZIF-8 and $\mathrm{Tb}_{2}(\mathrm{BDC})_{3}$, suggesting that $g$ can be a useful method for tailoring MOF morphology. Of note is that other MOFs may display different growth kinetics and trends in high and low- $g$, however these should be experimentally determined in a case-by-case basis. 

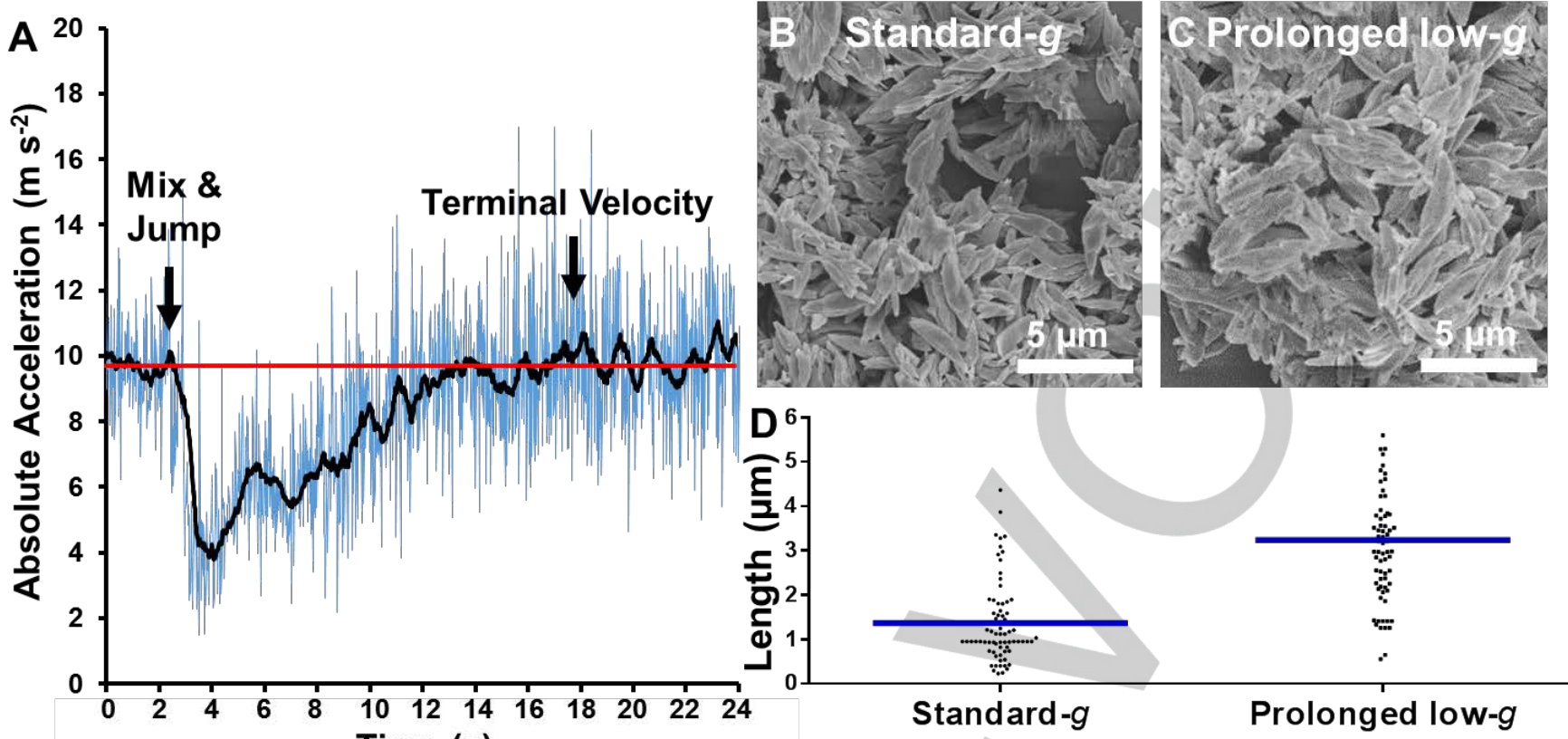

Time (s)

Figure 4. Effect of prolonged low-g $(\sim 15 \mathrm{~s})$ on $\mathrm{Tb}_{2}(\mathrm{BDC})_{3}$ crystallization. A, processed accelerometer data for the jump experiment showing the acceleration experienced at each time point. The red line represents $9.8 \mathrm{~m} \mathrm{~s}^{-2}(g=1)$. Note that time 0 corresponds with 1510 seconds in the raw data (Figure S11 and S12) SEM images of the $\mathrm{Tb}_{2}(\mathrm{BDC})_{3}$ MOFs prepared under standard- $g(\mathrm{~B})$ and prolonged low- $g(\mathrm{C})$. D, graph of average diameter (blue bar) and size distribution (black dots) depending on the $g$ applied during the synthesis. $\mathrm{Tb}_{2}(\mathrm{BDC})_{3}$ was prepared by mixing the precursors immediately before the authors jumped from a plane at an altitude of $\sim 4,000 \mathrm{~m}$ to generate prolonged low- $g$.

\section{Conclusions}

Three types of MOFs, namely HKUST-1, ZIF-8, and $\mathrm{Tb}_{2}(\mathrm{BDC})_{3}$ were grown under varied $g$. We observed that increasing $g$ results in smaller particle-size distributions, while decreasing $g$ results in larger particles. Furthermore, our results suggested that MOF crystal growth in low- $g$ was governed by facet oriented crystal fusion due to the reduced molecular transport of precursors in solution that would typically occur due to convection. Finally, unique experimental protocols were established for low-cost low- $g$ experimentation that should increase access to low- $g$ experiments for the MOF community, and also the crystal community at large. This study furthers our understanding of MOF crystallization and adds a new tool for controlling MOF morphology during synthesis.

\section{Experimental Section}

Materials. D,L-serine, 2-methylimidazole $(\mathrm{HmIm})$, and terbium chloride hexahydrate were purchased from Sigma Aldrich (Australia). Trimesic acid and copper nitrate trihydrate were purchased from Acros Organics (Australia). Zinc acetate dihydrate was obtained from Alfa Aesar (Australia). Disodium terephthalate was purchased from $\mathrm{TCl}$ (Australia). All other reactants were purchased from Sigma-Aldrich and used without further modification.

Synthesis of ZIF-8 crystals with serine. Serine $(1.3 \mathrm{mg})$ was dissolved in $\mathrm{Hm} / \mathrm{m}$ solution $(0.5 \mathrm{~mL}, 160 \mathrm{mM})$ in deionized water. This mixture solution was then combined with zinc acetate dihydrate solution $(0.5 \mathrm{~mL}$,
$40 \mathrm{mM}$ ) in deionized water. After shaking, the mixture solution was immediately subjected to varying $g$.

Synthesis of $\mathrm{Tb}_{2}(\mathrm{BDC})_{3}$ crystals. Aqueous solution of $\mathrm{TbCl}_{3} \cdot 6 \mathrm{H}_{2} \mathrm{O}(0.5$ $\mathrm{mL}, 20 \mathrm{mM}$ ) was mixed with aqueous solution of disodium terephthalate (BDC, $0.5 \mathrm{~mL}, 20 \mathrm{mM}$ ). After shaking, the mixture was immediately subjected to varying $g$.

Synthesis of HKUST-1 crystals. HKUST-1 was prepared by dissolving the precursors in ethanol, and then $\mathrm{Cu}\left(\mathrm{NO}_{3}\right)_{2} \cdot 3 \mathrm{H}_{2} \mathrm{O}(250 \mu \mathrm{L}, 200 \mathrm{mM})$ and BTC $(250 \mu \mathrm{L}, 133 \mathrm{mM})$ were added to $500 \mu \mathrm{L}$ of $50 \%$ ethanol for a final volume of $1 \mathrm{~mL}$ of solution with a final solvent ratio of $25 \%$ water / $75 \%$ ethanol. After shaking, the mixture was immediately subjected to varying $g$.

MOF washing. The obtained precipitate for all MOFs was centrifuged (5,000-10,000 RCF) and washed three times with fresh ethanol.

Centrifugation experiments. High- $g$ was generated by placing the justmixed MOF solution in a centrifuge at 20,50 , or 100 RCF.

Drop experiment. Drop experiments were performed from the roof of New Horizons building (c.a. $25 \mathrm{~m}$ from ground) at Monash University (Clayton - Melbourne). The precursor solutions were mixed in a microcentrifuge tube $(1.5 \mathrm{~mL})$ engineered with a soft rubber cushion and immediately dropped from the roof of ( $4^{\text {th }}$ floor). The samples were left to incubate for a total of $10 \mathrm{~min}$ after mixing before washing

Skydiving jump experiment. Although there is some controversy surrounding the life-saving potential of parachutes, ${ }^{[26]}$ it was decided that they would be a safe way to increase drag and reduce velocity after the prolonged low- $g$ portion of the skydiving experiments. Tandem skydiving 
was performed in quadruplicate with the mass of the tandem group kept constant in the $140-150 \mathrm{~kg}$ range. A plane was flown up to $\sim 4,000 \mathrm{~m}$ at which point the tandem groups exited the plane within $\sim 10 \mathrm{~s}$ of each other. Immediately before exiting the plane, the home-built injectors carrying the MOF precursor solutions was used to mix the solutions (see Video S1). After $\sim 55 \mathrm{~s}$ of freefall and terminal velocity, the parachute was deployed followed by $\sim 7 \mathrm{~min}$ of descent to the ground. The samples were left to incubate for a total of $10 \mathrm{~min}$ after mixing, meaning that after landing the samples were almost immediately taken to the mobile lab for washing.

Accelerometer studies. To record the $g$-force experienced by the MOF solution during the free fall, an Android smart phone equipped with an accelerometer record app (Accelerometer Analyzer, Google Play Store) was either dropped from the roof (inside a soft foam case) or carried in a pocket during the skydive. The raw acceleration was recorded for $x, y$, and $z$. The corresponding $g$ (absolute acceleration) was calculated from the acceleration vectors as the absolute magnitude.

Characterization. Optical micrographs were obtained using an Olympus BX60M microscope. Scanning electron microscope (SEM) images of samples were taken on a Zeiss MERLIN SEM at an accelerating voltage of $5.0 \mathrm{kV}$. Synchrotron SAXS data were collected at the SAXS/WAXS beamline at the Australian Synchrotron. The scattered radiation was registered by the detector (Pilatus $1 \mathrm{M}$ ). DLS data was collected with a Malvern Zetasizer.

\section{Acknowledgements}

We acknowledge the Australian Synchrotron for beamline access, JJR and $\mathrm{KL}$ acknowledge the Office of the Chief Executive of CSIRO for funding their fellowships, and MB acknowledge the Australian Government for funding through an Australian Postgraduate Award. MB, MF and JG acknowledge the ARC Centre of Excellene in Convergent Bio-Nano Science and Technology.

\section{Supporting Information}

Supporting Information containing additional data, and raw accelerometer readings can be found online.

Keywords: crystallization • porous coordination polymer • biomimetic $\cdot$ gravitational force $\cdot$ microgravity

[1] P. Falcaro, R. Ricco, C. M. Doherty, K. Liang, A. J. Hill, M. J. Styles, Chem. Soc. Rev. 2014, 43, 5513-5560.

[2] a) K. Sumida, D. L. Rogow, J. A. Mason, T. M. McDonald, E. D. Bloch Z. R. Herm, T.-H. Bae, J. R. Long, Chem. Rev. 2011, 112, 724-781; b) M. P. Suh, H. J. Park, T. K. Prasad, D.-W. Lim, Chem. Rev. 2011, 112, 782-835.

[3] J.-R. Li, J. Sculley, H.-C. Zhou, Chem. Rev. 2011, 112, 869-932.

[4] M. Yoon, R. Srirambalaji, K. Kim, Chem. Rev. 2011, 112, 1196-1231.

[5] L. E. Kreno, K. Leong, O. K. Farha, M. Allendorf, R. P. Van Duyne, J. T. Hupp, Chem. Rev. 2011, 112, 1105-1125.

[6] S. Bureekaew, S. Horike, M. Higuchi, M. Mizuno, T. Kawamura, D. Tanaka, N. Yanai, S. Kitagawa, Nat. Mater. 2009, 8, 831-836.

[7] Y. Cui, Y. Yue, G. Qian, B. Chen, Chem. Rev. 2011, 112, 1126-1162.
[8] a) K. Liang, R. Ricco, C. M. Doherty, M. J. Styles, S. Bell, N. Kirby, S Mudie, D. Haylock, A. J. Hill, C. J. Doonan, P. Falcaro, Nat. Commun. 2015, 6, 7240; b) P. Horcajada, T. Chalati, C. Serre, B. Gillet, C. Sebrie, T. Baati, J. F. Eubank, D. Heurtaux, P. Clayette, C. Kreuz, Nat. Mater 2010, 9, 172-178.

[9] a) P. Falcaro, R. Ricco, A. Yazdi, I. Imaz, S. Furukawa, D. Maspoch, R. Ameloot, J. D. Evans, and C. J. Doonan, Coord. Chem. Rev. 2016, 307, 237-254. b) Q.-L. Zhu, Q. Xu, Chem. Soc. Rev. 2014, 43, 5468-5512.

[10] a) R. Ricco, K. Konstas, M. J. Styles, J. J. Richardson, R. Babarao, K. Suzuki, P. Scopece, P. Falcaro, J. Mater. Chem. A 2015, 3, 19822 19831; b) S.-H. Huo, X.-P. Yan, Analyst 2012, 137, 3445-3451.

[11] a) W. Morris, C. J. Doonan, O. M. Yaghi, Inorg. Chem. 2011, 50, 6853 6855 ; b) K. K. Tanabe, S. M. Cohen, Chem. Soc. Rev. 2011, 40, 498 519; c) Z. Wang, S. M. Cohen, Chem. Soc. Rev. 2009, 38, 1315-1329.

[12] K. Liang, C. Carbonell, M. J. Styles, R. Ricco, J. Cui, J. J. Richardson, D. Maspoch, F. Caruso, P. Falcaro, Adv. Mater. 2015, 27, 7293-7298.

[13] N. Stock, S. Biswas, Chem. Rev. 2011, 112, 933-969.

[14] K. Li, D. H. Olson, J. Seidel, T. J. Emge, H. Gong, H. Zeng, J. Li, J. Am. Chem. Soc. 2009, 131, 10368-10369.

[15] S. Sacanna, D. J. Pine, G.-R. Yi, Soft Matter 2013, 9, 8096-8106.

[16] a) Y. Pan, D. Heryadi, F. Zhou, L. Zhao, G. Lestari, H. Su, Z. Lai, CrystEngComm 2011, 13, 6937-6940; b) Y. Zhao, J. Zhang, B. Han, J. Song, J. Li, Q. Wang, Angew. Chem. Int. Edit. 2011, 50, 636-639.

[17] K. Liang, R. Ricco, C. M. Doherty, M. J. Styles, P. Falcaro, CrystEngComm 2016, In Press.

[18] a) J. A. Bohrman, M. A. Carreon, Chem. Commun. 2012, 48, 5130 5132; b) P. Falcaro, F. Normandin, M. Takahashi, P. Scopece, H Amenitsch, S. Costacurta, C. M. Doherty, J. S. Laird, M. D. Lay, F. Lisi, Adv. Mater. 2011, 23, 3901-3906

[19] M. Hu, S. Furukawa, R. Ohtani, H. Sukegawa, Y. Nemoto, J. Reboul, S Kitagawa, Y. Yamauchi, Angew. Chem. 2012, 124, 1008-1012.

[20] a) J.-F. Chen, Y.-H. Wang, F. Guo, X.-M. Wang, C. Zheng, Ind. Eng. Chem. Res. 2000, 39, 948-954; b) A. Lenhoff, P. Pjura, J. Dilmore, T. Godlewski, J. Cryst. Growth 1997, 180, 113-126; c) M. C. Hales, T. A Steinberg, W. N. Martens, J. Non-Cryst. Solids 2014, 396, 13-19; d) A Vergara, B. Lorber, A. Zagari, R. Giegé, Acta. Crystallogr. D 2003, 59, 2-15.

[21] M. Hu, J.-S. Jiang, CrystEngComm 2010, 12, 3391-3393.

[22] K. S. Park, Z. Ni, A. P. Côté, J. Y. Choi, R. Huang, F. J. Uribe-Romo, H. K. Chae, M. O'Keeffe, O. M. Yaghi, P. Natl. Acad. Sci. USA 2006, 103 10186-10191.

[23] S. S.-Y. Chui, S. M.-F. Lo, J. P. Charmant, A. G. Orpen, I. D. Williams, Science 1999, 283, 1148-1150.

[24] T. M. Reineke, M. Eddaoudi, M. Fehr, D. Kelley, O. Yaghi, J. Am. Chem. Soc. 1999, 121, 1651-1657.

[25] S. Ostrach, Annu. Rev. Fluid Mech. 1982, 14, 313-345.

[26] G. C. Smith, J. P. Pell, BMJ 2003, 327, 1459.

[27] J. M. DiFrancesco, J. M. Olson, npj Microgravity 2015, 1, 15001.

[28] J. Cravillon, R. Nayuk, S. Springer, A. Feldhoff, K. Huber, M. Wiebcke, Chem. Mater. 2011, 23, 2130-2141.

[29] a) E. V. Shevchenko, D. V. Talapin, H. Schnablegger, A. Kornowski, Ö. Festin, P. Svedlindh, M. Haase, H. Weller, J. Am. Chem. Soc. 2003 125, 9090-9101; b) M. Oh, C. A. Mirkin, Nature 2005, 438, 651-654.

[30] J. P. Patterson, P. Abellan, M. S. Denny Jr, C. Park, N. D. Browning, S M. Cohen, J. E. Evans, N. C. Gianneschi, J. Am. Chem. Soc. 2015, 137, 7322-7328.

[31] R. Koppram, E. Tomás-Pejó, C. Xiros, L. Olsson, Trends Biotechnol. 2014, 32, 46-53.

[32] J. Zhu, M. Li, R. Rogers, W. Meyer, R. Ottewill, W. Russel, P. Chaikin, Nature 1997, 387, 883-885

[33] D. Feng, K. Wang, Z. Wei, Y.-P. Chen, C. M. Simon, R. K. Arvapally, R. L. Martin, M. Bosch, T.-F. Liu, S. Fordham, Nat. Commun. 2014, 5 5723

[34] D. Zacher, J. Liu, K. Huber, R. A. Fischer, Chem. Commun. 2009, 9, 1031-1033. 


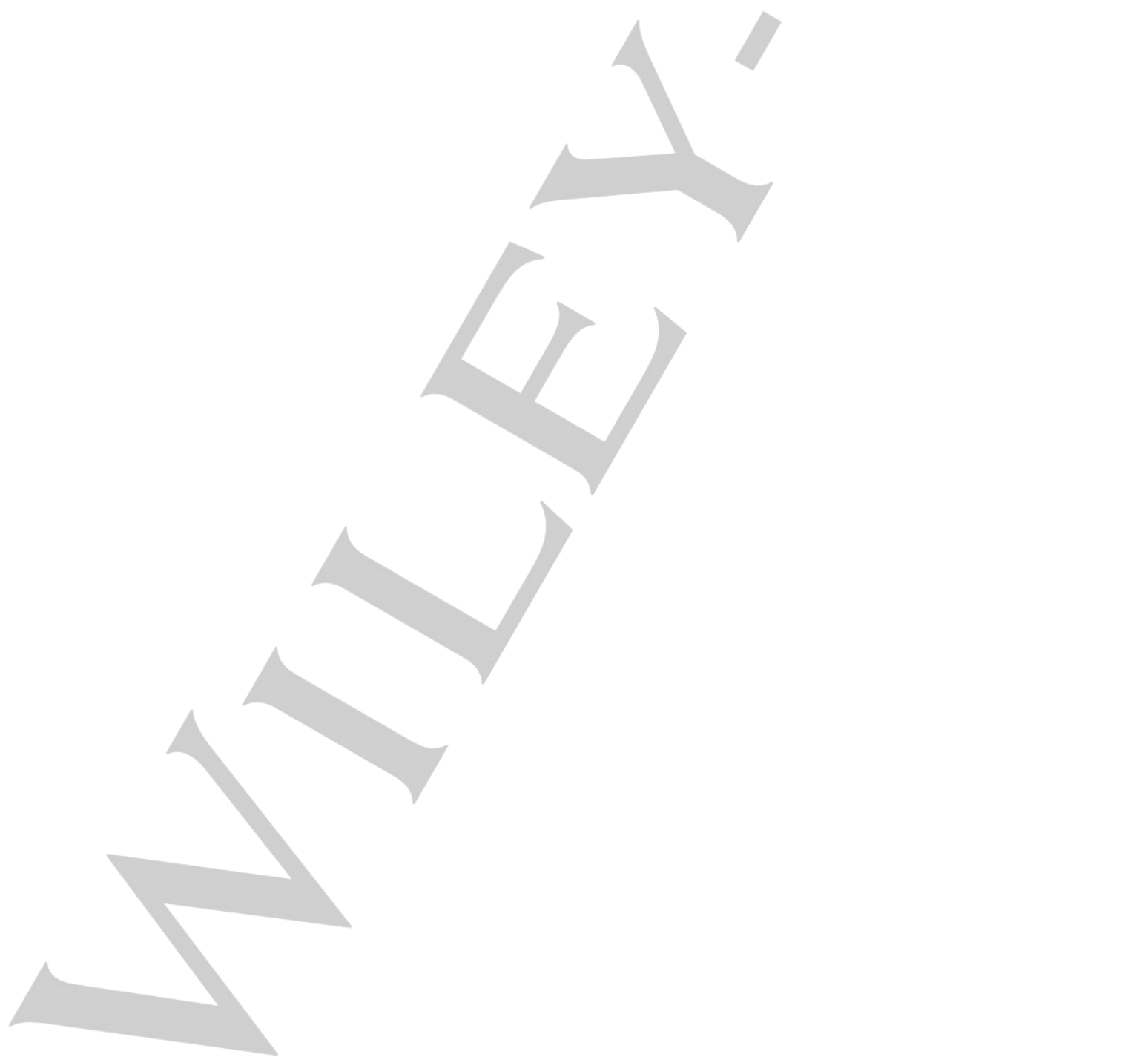




\section{Entry for the Table of Contents}

\section{FULL PAPER}

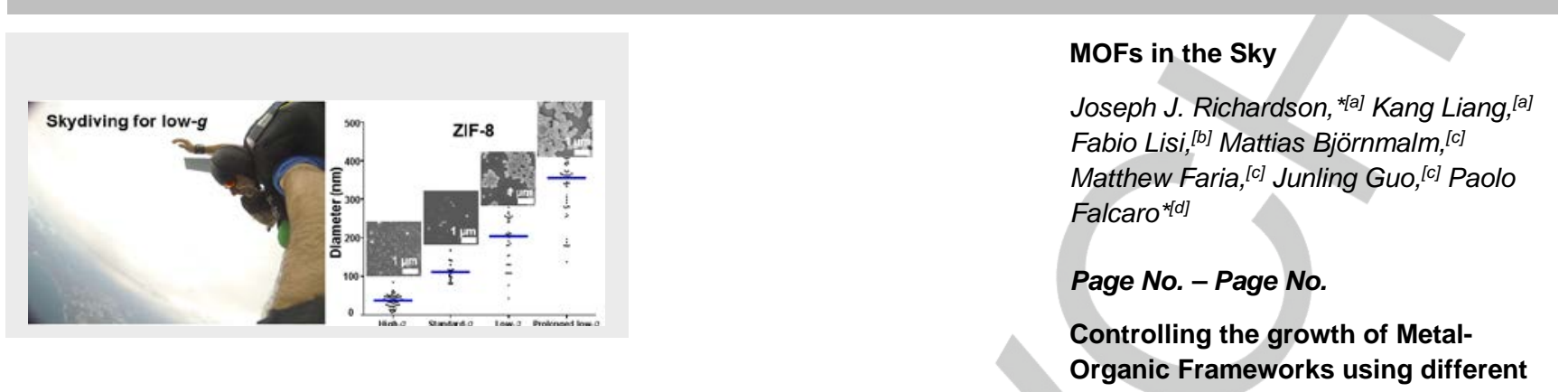

Metal-Organic Frameworks (MOFs) are grown under different gravitational-forces. We demonstrate that larger MOFs grow at lower $g$ force $(g<1)$, while smaller MOFs grow at higher $g$-force $(g=20)$. This data matches well with historical studies on crystal growth in microgravity, suggesting that the MOFs explored herein are governed by similar growth mechanisms to a wide variety of other crystals. 